\title{
Rapid onset transverse myelitis in adolescence: implications for pathogenesis and prognosis
}

\author{
Jo M Wilmshurst, Matthew C Walker, Keith R E Pohl
}

\begin{abstract}
Five adolescents with transverse myelitis were reviewed. All presented with a rapid onset paralysis of the lower limbs and impairment of bladder control. The maximum disability developed between 10 minutes and six hours. There was no history of trauma, asthma, or prodromal illness. Investigations failed to demonstrate a vascular cause. Extensive spinal cord abnormalities were observed on magnetic resonance imaging. Electrophysiological investigations, performed in four cases, were all consistent with anterior horn cell damage. In all five adolescents there was poor recovery. The underlying pathogenesis of this rapid onset condition remains a subject of debate. Similarities with both transverse myelitis and fibrocartilaginous emboli are evident, widening the spectrum of conditions within the transverse myelitis umbrella. These observations suggest that in rapid onset "transverse myelitis" the combination of extensive hyperintensity on spinal cord neuroimaging with electrophysiological evidence of anterior horn cell involvement might have adverse prognostic significance.

(Arch Dis Child 1999;80:137-142)
\end{abstract}

Keywords: transverse myelitis; fibrocartilaginous emboli; Hopkins's syndrome; adolescence; pathogenesis; prognosis

Transverse myelitis is a well recognised rare condition that can occur in childhood and adolescence. ${ }^{12}$ Onset is usually associated with severe back pain followed by paralysis. ${ }^{2} \mathrm{Al}-$ though it is an acute condition, evolving over one to three weeks, the speed of onset of symptoms is rarely as rapid as was seen in our patients. In the early stages, there is lower limb flaccid paralysis, with a sensory level commonly in the mid-thoracic area. It is often possible to show dissociated sensory loss, with pain, temperature, and light touch sensation being affected, but joint position and vibration well preserved. ${ }^{1}$ Sphincter involvement is common. The flaccidity gradually changes to spasticity with the development of upper motor neurone signs. Patients may have several levels of the spinal cord affected with a mixed sensory and motor picture, but clinical evidence of anterior horn cell damage is not a recognised feature. ${ }^{3}$ Imaging might be normal or abnormal, the abnormalities consisting of either localised swelling or hyperintensity of the cord at the affected levels. ${ }^{4-7}$ The aetiology of the inflammatory response of transverse myelitis is unknown. Postinfective, autoimmune, and primary inflammatory mechanisms have been postulated. A vascular basis has long been suggested.

The condition fibrocartilaginous emboli causes more rapid paralysis than usually occurs with transverse myelitis, with onset over minutes to hours. ${ }^{8}$ Since the 1960 s it has been recognised that fibrocartilaginous emboli from the nucleus pulposus of the intervertebral disc can cause a fatal acute anterior spinal artery syndrome. Most cases of fibrocartilaginous emboli described are of sudden onset paralysis at a cervical level following some minor trauma. Many cases of transverse myelitis might be a result of fibrocartilaginous emboli, but the diagnosis of fibrocartilaginous emboli without postmortem data is unreliable. ${ }^{9}$

Conditions of paralysis in which anterior horn cell damage is a more specific pathological finding include poliomyelitis ${ }^{10}$ and Hopkins's syndrome (acute postasthmatic amyotrophy). ${ }^{11}$ These are discussed in relation to our patients.

The overall prognosis in childhood transverse myelitis is often remarkably good and recovery is often rapid and complete. ${ }^{12} \mathrm{We}$ outline how our five patients highlight the difficulties of diagnosing acute onset paralysis and how the poor outcome of our patients suggests relevant prognostic indicators.

\section{Patient 1}

A previously well, 141/2 year old girl, vaccinated against poliomyelitis as an infant, developed paralysis of her lower limbs during a period of half an hour. She had no prodromal features. She described throwing a small bag of rubbish over her right shoulder, developing a "wobbly" feeling in her knees and staggering to sit down. She had severe pain in her lower back and epigastrium for half an hour. On admission she was alert and orientated. Her lower limb deep tendon and abdominal reflexes were absent. Power was graded on Medical Research Council (MRC) scale at a score of $0 / 5$ in both legs. From the sensory level of T10 downwards light touch, pain, and temperature awareness were absent, vibration and proprioception were impaired. She required intermittent catheterisation for complete bladder emptying. Upper motor neurone features evolved, by six weeks minimal improvement was seen, and six months after presentation she remained in a wheelchair, and catheter dependent. Investigations failed to demonstrate any haematological or biochemical disturbance in the first and sixth weeks after presentation. Acute phase 


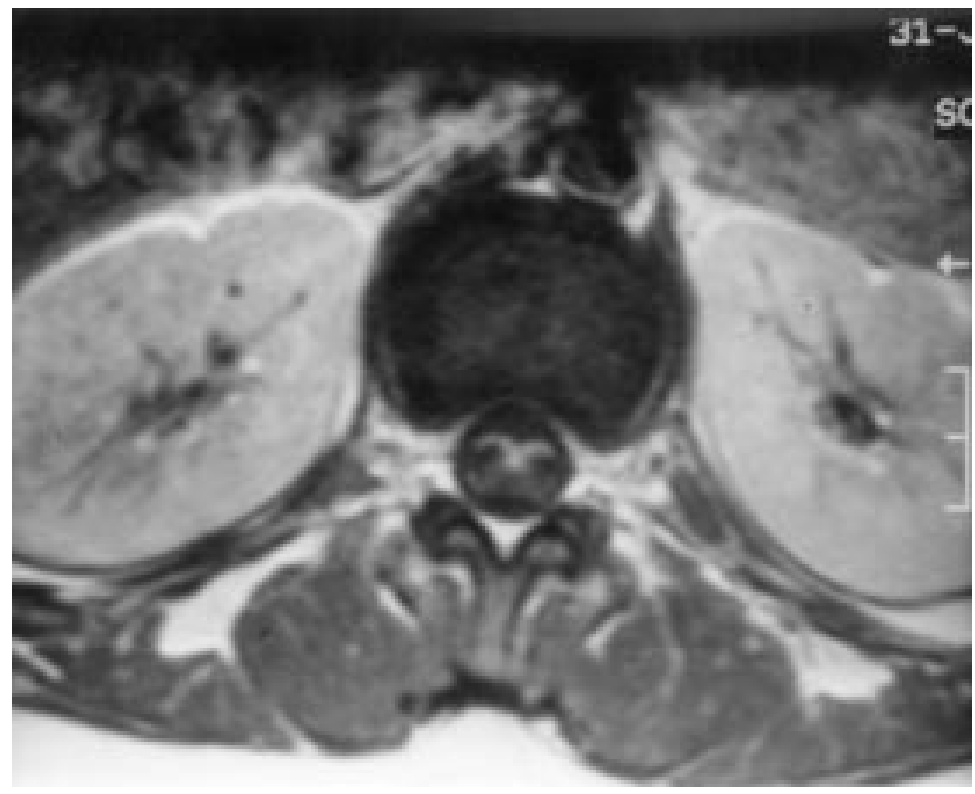

Figure 1 Magnetic resonance imaging of the spinal cord; $T$ weighted axial image, demonstrating anterior grey matter high signal in region of the anterior horns in patient 1.

reactants were normal. Procoagulant studies and serum B12 concentrations were normal. Serum, throat swab, and stool samples failed to show any viral infection. Cerebrospinal fluid (CSF) measurements performed on two occasions were unremarkable, apart from CSF protein rising from $0.5 \mathrm{~g} / 1$ in the first week to $0.9 \mathrm{~g} / 1$ at six weeks. No white blood cells were present. Polymerase chain reaction (PCR) analysis for enteroviruses in the CSF was negative. Urodynamic studies confirmed a neuropathic bladder.

Electromyography (EMG) and nerve conduction studies were performed at six weeks and two and a half months after paralysis. Initially, at six weeks, upper and lower limb sensory responses were normal. Upper limb motor study was normal. The lower limb responses demonstrated normal motor conduction velocities but slightly prolonged $\mathrm{F}$ wave latencies. Motor action potential amplitudes were reduced significantly in the lower limbs. There was evidence of active denervation in the lower limbs and lumbar paraspinal muscles, with no evidence of any motor unit potentials under volitional control. Frequent fibrillations were seen in the lower limbs. At two and a half months, the presence of $\mathrm{H}$ reflexes in the foot muscles was demonstrated, suggesting an upper motor neurone lesion. However, severe denervation persisted in myotomes of at least L3 to S1. There was asymmetry at the level of $\mathrm{S} 1$, with a much less affected gastrocnemius on the right. The picture was felt to be more compatible with a transverse myelitis with fallout of the anterior horn cells in affected segments.

Magnetic resonance imaging (MRI) of the spine at one week showed enhancement of the roots of the cauda equina with no evidence of any vascular anomaly. A repeat scan at three months demonstrated a linear area of altered signal in the anterior portion of the distal cord, extending up to the level of T9. This

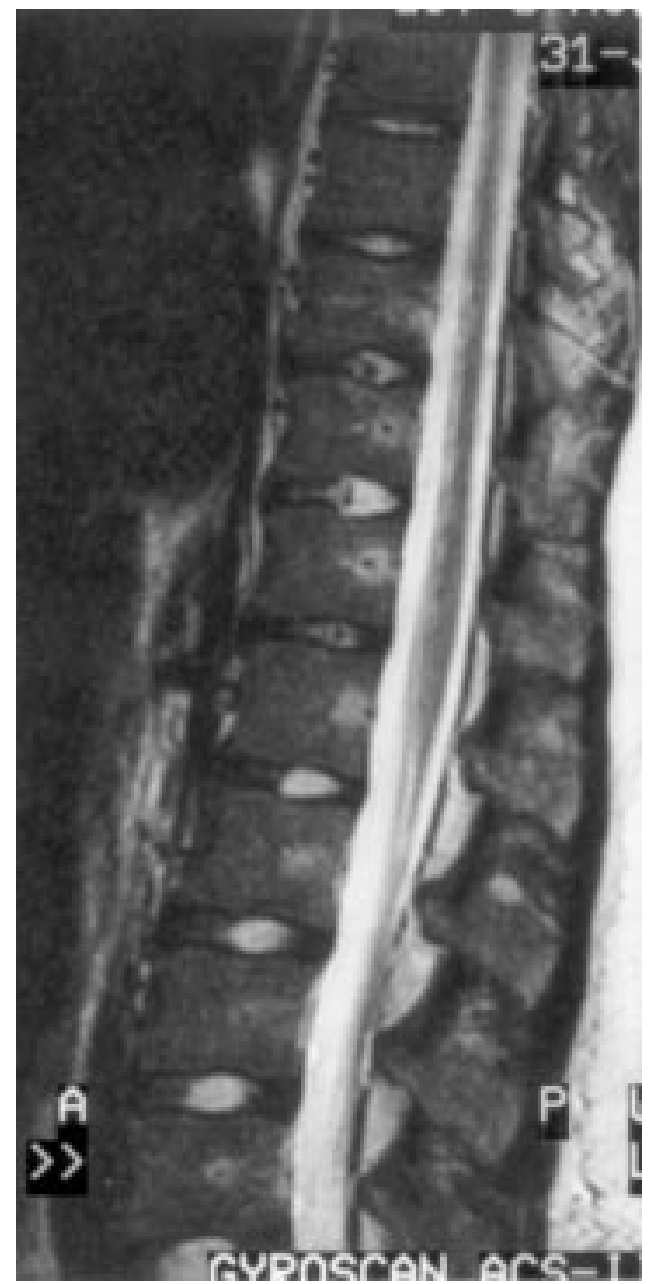

Figure 2 Magnetic resonance imaging of the spinal cord; $T$, weighted sagittal image, demonstrating linear area of anterior cord hyperintensity with associated Schmorl's nodes and disc degeneration in patient 1.

corresponded to the anterior horns, with enhancement of anterior roots in a symmetrical distribution (fig 1). No further enhancement of these lesions occurred after gadolinium administration. No evidence for a vascular aetiology was seen, but her neuroimaging did demonstrate evidence of intervertebral disc disease between $\mathrm{T} 12$ and $\mathrm{L} 1$, and the presence of Schmorl's nodes (fig 2).

\section{Patient 2}

A previously well, 15 year old girl, fully immunised against poliomyelitis, presented with a six hour history of evolving lower limb weakness. She had complained of mild lumbar back pain over the previous few days. On the morning of admission she had walked without a problem, over the day, she developed a band of discomfort around her epigastrium associated with back pain. Increasing lower limb weakness resulted in complete paralysis from the waist downwards.

On examination, no abnormality was detected above a sensory level of T9. Below this level there was complete paralysis, initially both motor and sensory, with absent deep tendon reflexes. Over the following three months improvement occurred, she could walk five 
Table 1 Summary of the five patients

\begin{tabular}{|c|c|c|c|c|c|c|c|}
\hline $\begin{array}{l}\text { Patient/ } \\
\text { sex }\end{array}$ & $\begin{array}{l}\text { Age } \\
\text { (years) }\end{array}$ & Rate of onset & $\begin{array}{l}\text { Sensory } \\
\text { level }\end{array}$ & $M R I$ & $\begin{array}{l}\text { Disc } \\
\text { disease }\end{array}$ & Nerve conduction studies/EMG & Outcome \\
\hline $1 / \mathrm{F}$ & $14^{1 / 2}$ & 30 minutes & $\mathrm{T} 10$ & Anterior cord hyperintensity $\mathrm{T} 9$-conus & Yes & Anterior horn cell fallout & Poor \\
\hline $2 / \mathrm{F}$ & 15 & 6 hours & T9† & $\begin{array}{l}\text { Anterior and right posterior cord } \\
\text { hyperintensity; distal thoracic cord }\end{array}$ & No & $\begin{array}{l}\text { Anterior horn cell fallout left }> \\
\text { right; most severe L3-S1 }\end{array}$ & $\begin{array}{l}\text { Moderate left leg most } \\
\text { weak }\end{array}$ \\
\hline $3 / \mathrm{M}$ & 14 & 30 minutes & L1 & $\begin{array}{l}\text { Hyperintensity anterior distal thoracic cord; } \\
\text { right }>\text { left }\end{array}$ & No & $\begin{array}{l}\text { Anterior horn cell fallout L5-S1; } \\
\text { right }>\text { left }\end{array}$ & $\begin{array}{l}\text { Moderate right leg most } \\
\text { weak }\end{array}$ \\
\hline $4 / \mathrm{M}$ & 16 & 10 minutes & T6/7 & Hyperintensity anterior cord T3-6; left > right & Yes & $\begin{array}{l}\text { Early evidence of anterior horn } \\
\text { cell fallout }\end{array}$ & Moderate \\
\hline $5 / \mathrm{F}$ & 15 & $\begin{array}{l}\text { "Seconds to } \\
\text { minutes" }\end{array}$ & $\mathrm{T} 11$ & $\begin{array}{l}\text { Hyperintensity anterior-central cord } \\
\text { T9-conus }\end{array}$ & Yes & Not done & Poor \\
\hline
\end{tabular}

Outcome: poor, wheelchair dependent; moderate, walking but residual gait impairment.

$\star$ Pain/temperature/light touch sensory involvement; tpatient 2 also had right posterior column involvement.

steps with support, but at six months she remained wheelchair dependent for community mobility. Although pain and temperature awareness gradually improved, the sensory abnormalities persisted, with an area of hypersensitivity over the right thigh in the L $2 / 3$ dermatome, with some impaired proprioception. Bladder control remained impaired.

All infective, autoimmune, and vascular investigations were unremarkable. Peripheral electrophysiology (EMG and nerve conduction studies) demonstrated evidence of an asymmetrical axonal degeneration in the lower limbs. No activity was present in the left lower leg but, in the left thigh, voluntary motor unit potentials were present, indicating recent vigorous sprouting. The right thigh was partly denervated, but to a lesser extent than the left. There was evidence of axonal fallout from at least L3 to S1. Significant fibrillations and sharp waves suggested anterior horn cell pathology. Sensory action potentials were normal.

MRI of the spine one week after presentation revealed minimal expansion of the distal cord with increased signal, most prominent in the anterior and the right posterior portion of the cord. There was no evidence of intervertebral disc disease.

\section{Patient 3}

A previously well, 14 year old boy on a school trip from Slovenia complained of a mild lower back pain overnight. There was no history of asthma or trauma, and he was fully immunised against poliomyelitis. The following day he developed a rapid onset paralysis of his lower limbs evolving over half an hour. He had a level of sensory loss from L1 downwards, with associated bladder involvement. He was initially areflexic. Over the following two weeks limited recovery occurred. This was asymmetric with some improvement in the left leg, but minimal recovery in the right leg. Sensation improved with some residual impairment of pain and temperature awareness and an area of hypersensitivity over dermatome L3 on the left. Bladder function remained impaired. Written reports from Slovenia at six months after presentation suggested little further recovery. Investigations revealed no evidence of infective, immunological, or vascular disease. Peripheral electrophysiology (EMG and nerve conduction studies) were performed just before his return to Slovenia at two weeks. At this stage, there was no evidence of fibrillation and absent $\mathrm{F}$ waves were noted, suggesting radicular or anterior horn cell abnormalities. At 10 weeks after paralysis, repeat peripheral electrophysiology (in Slovenia) demonstrated severe denervation of L5, S1, and S2. Complete denervation was apparent on the right side, with signs of reinnervation on the left. Sensory potentials were normal. At six months after paralysis, he remained wheelchair dependent for community mobility and still had bladder instability.

Abnormalities on MRI of the spine, one day after paralysis, consisted of an altered signal in the distal thoracic cord, with focal abnormalities within the anterior part of the cord-these were slightly more prominent on the right side. The appearances were felt to be consistent with an inflammatory process involving the distal cord. There was no evidence of intervertebral disc disease.

\section{Patient 4}

A previously well 16 year old boy described a minor upper respiratory tract infection from which he had fully recovered two weeks before presentation. On the day of presentation he had been physically active, but sustained no trauma. An hour after playing football he became aware of progressive weakness extending from his toes upwards in his left leg. Maximum paralysis occurred over 10 minutes. His right leg became affected over the following 12 hours. On admission, he had complete flaccid paralysis of his left leg, power in the right leg was graded 3/5 MRC. All lower limb deep tendon reflexes were absent or suppressed. Sensation was intact apart from alteration in pin prick sensation at dermatome T6/7. He developed urinary retention. Eight months after presentation he had persistent asymmetrical lower limb weakness and could walk with support. Investigations excluded infective, immunological, or vascular abnormalities. Peripheral electrophysiological investigations (EMG and nerve conduction studies) on the second day of his illness, performed on the lower limbs, demonstrated $\mathrm{F}$ waves which were of normal latency, but were difficult to elicit. MRI on the same day revealed widening of the thoracic cord from $\mathrm{T} 3$ to $\mathrm{T} 6$ and signal change within the cord, which was more prominent on the left side. No enhancement occurred with contrast medium and no abnormal vessels were seen. Disc dehydration was evident in the T8-11 region. The examination was repeated after a week and was unchanged. 
Table 2 Electrophysiological and neuroimaging findings in conditions suggested to involve anterior horn cells compared with patients 1, 2, and 3

\begin{tabular}{|c|c|c|c|c|c|c|}
\hline & Patient 1 & Patient 2 & Patient 3 & $A T M^{7}$ & Hopkins's syndrome ${ }^{17}$ & Poliomyelitis $^{21}$ \\
\hline SAP & Normal & Normal & Normal & $\mathrm{N} / \downarrow$ & Normal & Normal \\
\hline $\mathrm{MCV}$ & Normal & $\downarrow$ Lower limbs & $\downarrow_{\text {Lower limbs }}$ & $\downarrow$ Severe cases & $\downarrow$ Severe cases & Normal \\
\hline CMAP & $\downarrow /-$ Lower limbs & $\downarrow$ Lower limbs & $\downarrow$ Lower limbs & $\downarrow /-$ & $\downarrow /-$ & $\downarrow$ \\
\hline F wave & Slightly prolonged & & & - & $-/ \mathrm{N}$ & Unknown \\
\hline $\mathrm{H}$ reflex & + (Foot muscles) & L3-S1 & L5, S1, S2 severe on right & + & + & Early feature \\
\hline Denervation & Severe L3-S1 & + & & + & + & + \\
\hline Fibrillation & + & & & & & \\
\hline MRI findings & $\begin{array}{l}\text { Hyperintensity of } \\
\text { anterior horn cells }\end{array}$ & $\begin{array}{l}\text { Hyperintensity of } \\
\text { anterior distal cord } \\
\text { and right posteriorly }\end{array}$ & $\begin{array}{l}\text { Hyperintensity in anterior } \\
\text { distal thoracic cord; right } \\
>\text { left }\end{array}$ & $\begin{array}{l}\text { Normal; cord swelling; } \\
\text { hyperintensity- diffuse or } \\
\text { localised to AHC }\end{array}$ & $\begin{array}{l}\text { Hyperintensity of } \\
\text { anterior horn cells }{ }^{16} 17\end{array}$ & $\begin{array}{l}\text { Hyperintensity of } \\
\text { anterior horn cells }{ }^{10}\end{array}$ \\
\hline
\end{tabular}

$\downarrow$, reduced; +, present; -, absent; AHC, anterior horn cells; CMAP, compound motor action potential; MCV, motor conduction velocity; SAP, sensory action potential; ATM, acute transverse myelitis.

Patient 5

A 15 year old girl presented with sudden onset paralysis of her lower limbs described as occurring over seconds to minutes. She was areflexic on arrival, with a sensory level for pain and temperature at $\mathrm{T} 11$, but with sparing of the dorsal column modalities. She was also in urinary retention. She made minimal recovery with gait impairment and bladder and bowel instability.

Investigation failed to reveal any vascular, immunological, or infective cause. She did not have peripheral electrophysiology performed. MRI demonstrated increased signal on $T_{2}$ weighted images in the anterior-central cord from $\mathrm{T} 9$ to the conus with no evidence of cord oedema. No enhancement occurred following contrast. Early dehydration change was seen in the L4/5 intervertebral disc and there was a small midline posterior disc protrusion at $\mathrm{L} 3 / 4$ and $L 4 / 5$. No evidence of neural compression was seen.

Table 1 summarises the main features of these five cases.

\section{Discussion}

Transverse myelitis is a rare condition in childhood. It is typically associated with pain, usually in the back, followed by the evolution of weakness (often asymmetrical), and bladder disturbance. Although generally an acute illness, all our cases had a very rapid onset with marked progression of symptoms. Such rapid onset of paralysis is reported to be a feature of poor long term prognosis. ${ }^{2}$

Prognosis for recovery in transverse myelitis is variable. In one study, 15 of 25 children made a good to moderate recovery ${ }^{1}$ and in another 12 of 21 made such a recovery. ${ }^{2}$ These figures are better than those for adults. Most patients are expected to make the majority of their recovery in the first three months after paralysis. ${ }^{2}$ The patients in our series are all in a poor outcome group.

Spinal cord MRI abnormalities are seen frequently in transverse myelitis. Mild fusiform swelling is the most frequently seen abnormality ${ }^{4}$; however, a wider range of abnormalities has been described, particularly hyperintensity of the anterior portion of the cord consistent with the anterior horns. ${ }^{6}{ }^{12}$ All our patients showed increased signal on $T_{2}$ weighted images, patients $1,3,4$, and 5 demonstrating hyperintensity especially in the anterior regions of the spinal cord.
Excluding our patient series, little information on the electrophysiological findings in acute transverse myelitis is available. A previous study in India found that all patients with poor outcome had evidence of anterior horn cell fallout. ${ }^{7}$ Patients 1-4 of our study group had electrophysiological changes consistent with anterior horn cell fallout. The most dramatic changes were in patient 1 , who was also the most severely affected of the group.

We propose that rapidly progressing transverse myelitis should be considered as a specific subgroup within the spectrum of the disease. Furthermore, it should be considered to be a feature associated with poor outcome. The demonstration on MRI imaging of hyperintensity on $\mathrm{T}_{2}$ weighted sequences in the anterior region of the spinal cord and electrophysiological evidence of anterior horn cell fallout are additional poor outcome factors.

The pathogenesis of transverse myelitis is poorly understood. Do the observations in our series of sudden onset transverse myelitis support any particular pathogenic theory? Our observation of anterior horn cell involvement in this rapid onset group of patients leads us to speculate that a wider spectrum of conditions might fall under the umbrella of transverse myelitis. Transverse myelitis is not a diagnosis, it is a condition of cord inflammation that occurs following a number of triggers (infective, inflammatory, and autoimmune) and is of unknown aetiology. It has been postulated that the origins of transverse myelitis might be an acute vascular process. ${ }^{1}$ There are many features in keeping with an anterior spinal artery injury - particularly with our rapid onset group. The blood supply to the anterior region of the cord is less extensive and thus more vulnerable than the posterior region. ${ }^{13}$ Compromise can arise after occlusion of a large radicular branch or a lesion of the aorta. However, despite detailed neuroimaging in our patients, we were unable to establish evidence of a vascular malformation.

Fibrocartilaginous emboli was an important differential in our patients. It is a rarely recognised but important condition of sudden onset paralysis usually described at the cervical level. ${ }^{8}{ }^{14}$ Most recognised cases died. Diagnosis is confirmed by specific postmortem staining for fibrocartilaginous emboli matter within the vascular supply of the necrotic cord, and cannot be made reliably without such pathology. This material can be scattered and difficult to locate. ${ }^{13}$ The condition followed a minor 
traumatic episode in over half the reported cases. A similar illness is widely recognised in veterinary medicine. ${ }^{15}$ There is agreement that the emboli originate from the intervertebral disc, but the mechanism whereby disc fragments enter the vessels is not yet understood. There are many theories as to how the emboli enter the circulation, one being that high axial loading causes increased pressure within an abnormally vascularised but not ruptured disc. Semi-fluid nucleus pulposus material injects into the small arteries, with retrograde spread to a radicular artery. The intervertebral disc is usually avascular, but vessels may persist until adolescence, or reappear later in life in degenerated discs. The alternative theory is that an intervertebral disc ruptures laterally, causing damage to an adjacent radicular artery, with consequent entrance of disc fragments into the arterial circulation. ${ }^{814}$ The presence of Schmorl's nodes is also felt to be important in the underlying pathology. Patients 1,4 , and 5 in our study had disc erosion and patient 1 also had Schmorl's nodes, but the remaining two children did not. External trauma was not a likely trigger in any of the children.

Diagnosis of fibrocartilaginous emboli is usually made on postmortem examination. This is more likely to occur with paralysis at the cervical level. Because most patients with lumbar level paralysis survive, a pathological review is not normally possible. One case has been described in whom analysis was performed after surgical intervention. This confirmed the presence of fibrocartilaginous emboli within the vasculature of the cord. ${ }^{13}$

One documented case of fibrocartilaginous emboli in a 16 year old girl demonstrated hyperintensity of the cord on neuroimaging, along with denervation on electrophysiology, as can be seen in cases of acute transverse myelitis. The clinical picture of transverse myelitis closely resembles fibrocartilaginous emboli; thus, it can be difficult to separate the conditions without pathological evidence. It is possible that after a drop in vascular pressure, any area of poorly perfused, compromised cord is associated with fibrocartilaginous emboli leakage and consequent backflow of such emboli into the circulation. It should be remembered that the emboli are easily missed without pathological examination.

Greater understanding is needed of the pathogenesis of rapid onset paralysis, early attempts at reperfusion might alter the outcome in such cases.

There are a number of other rare paediatric conditions noted for their anterior horn cell involvement, Hopkins's syndrome is an example. Table 2 summarises the peripheral electrophysiological and neuroimaging findings recognised in transverse myelitis and related conditions. Hopkins's syndrome is a poliomyelitis-like illness of unknown aetiology, resulting in a flaccid paralysis of an extremity occurring during the recovery phase of an asthmatic attack of variable severity. ${ }^{11}$ Investigations in Hopkins's syndrome show many similarities with our group of patients. The age

\section{Key messages}

- Rapid onset of paralysis (less than six hours), extensive hyperintensity on spinal cord $\mathrm{T}_{2}$ weighted $\mathrm{MRI}$, and anterior horn cell involvement are associated with poor outcome in adolescents

- Transverse myelitis is a condition involving a spectrum of disease; the cause of paralysis should be questioned. Vascular impairment secondary to fibrocartilaginous emboli should be considered

- In making the diagnosis of Hopkins's syndrome, the pathogenic spectrum of transverse myelitis should be considered

range of described cases is between 13 months and 12 years, poliomyelitis has been excluded in all, and permanent paralysis is typical. Onset is over a day or two, with few cases over a number of hours. The few reports of neuroimaging in Hopkins's syndrome suggest consistently abnormal findings in the anterior part of the spinal cord on $T_{2}$ weighted sequences, ${ }^{16}{ }^{17}$ as is seen in poliomyelitis. ${ }^{10}$ Electrophysiology in Hopkins's syndrome demonstrates an abnormality consistent with anterior horn cell damage. Although this condition has been labelled a lower motor neurone abnormality, two cases described by Hopkins ${ }^{11}$ and a further case $^{18}$ had brisk reflexes and extensor plantars. In 1975, Danta suggested that the underlying radiculopathy might extend to involve the cord. ${ }^{19}$

A non-asthmatic 7 year old boy with a viral illness, who developed a poliomyelitis-like paralysis of both arms, was reported as suffering a Hopkins-like illness. ${ }^{20}$ PCR analysis of CSF demonstrated herpesvirus type 1 . The patient had acute cervical cord damage affecting both anterior horn cell and sensory nerve roots. On MRI, there was widening in the upper half of the cervical cord and a signal increase from $\mathrm{T}_{2}$ weighted images.

Thus, Hopkins's syndrome and transverse myelitis have features in common. Excluding the association with asthma, there are many similarities reinforcing the inclusion of Hopkins's syndrome under the transverse myelitis umbrella.

\section{Conclusion}

Prognosis of rapid onset paralysis in adolescence can be assessed by a combination of rate of onset (within 12 hours), the extent of neuroimaging intensity, and anterior horn cell involvement. The possibility of fibrocartilaginous emboli needs to be considered when investigating this group of patients. Relatively sudden anterior horn cell disease appears to be a pathologically irreversible feature of a range of paediatric conditions, suggesting a common pathogenic mechanism.

The authors thank Dr J Payan, Dr MD O'Brien, Professor RAC Hughes, Dr T Cox, Dr R Howard, and Dr F Schon for thei involvement with these patients and their valuable opinions. 
1 Paine RS, Byers RK. Transverse myelopathy in childhood. Am f Dis Child 1953;85:51-163.

Dunne K Hopkins IJ Shield LK Acute transverse myelopathy in childhood. Dev Med Child Neurol 1986;28: 198-204.

3 Berman M, Feldman S, Alter M, Zilber N, Kahana E. Acute transverse myelitis: incidence and aetiologic considerations. Neurology 1981;31:966-7

4 Marine D, Wang H, Kumar AJ, Zinreich SJ, Rosenbaum AE. CT myelography and MR imaging of acute transverse myelitis. F Comput Assisted Tomogr 1987;11:606-8.

5 Shen WC, Lee SK, Ho YJ, Lee KR, Mak SC, Chi CS. MRI of sequela of transverse myelitis. Paediatr Radiol 1992;22: 382-3.

6 Tartaglino LM, Croul SE, Flanders AE, et al. Idiopathic acute transverse myelitis: MR imaging findings. Radiology 1996;201:661-9.

7 Misra UK, Kalita J, Kumar S. A clinical, MRI and neurophysiological study of acute transverse myelitis. $\mathcal{F}$ Neurol Sci 1996;138:150-6.

8 Tosi L, Rigoli G, Beltramello A. Fibrocartilaginous embolism of the spinal cord: a clinical and pathological lism of the spinal cord: a clinical and pathological

9 Bots G, Th AN, Wattendorff AR, Buruma OJS, Roos RAC, Endtz LJ. Acute myelopathy caused by fibrocartilaginous emboli. Neurology 1981;31:1250-6.

10 Malzberg MS, Rogg JM, Tate CA, Zayas V, Easton JD Poliomyelitis: hyperintensity of the anterior horn cells in MR images of the spinal cord. Am $\mathcal{F}$ Radiol 1993;161:863-5.

11 Hopkins IJ. A new syndrome: poliomyelitis-like illness associated with acute asthma in childhood. Australian Paediatric fournal 1974;10:273-6.
12 Sanders KA, Khandji A, Mohr JP. Gadolinium-MRI in acute transverse myelopathy. Neurology 1990;40:1614-16.

13 Scully RE, Eugene JM, McNeely WF, McNeely BU. Case records of the Massachusettes General Hospital (case 5-1991). N Engl f Med 1991;324:322-32.

14 Toro G, Roman GC, Navarro-Roman L, Cantillo J, Serrano B, Vergara I. Natural history of spinal cord infarction caused by nucleus pulposus embolism. Spine 1994 19:3606.

15 Cook JR Jr. Fibrocartilaginous emboli. Vet Clin North Am Small Anim Pract 1988;18:581-92.

16 Arita J, Nakae Y, Matsushima H, Maekawa K. Hopkins syndrome: $T_{2}$-weighted high intensity of anterior horn on spinal MR imaging. Pediatr Neurol 1995;13:263-5.

17 Liedholm LJA, Eeg-Olofsson O, Ekenberg BEK, Nicolaysen $\mathrm{RB}$, Torbergsen T. Acute postasthmatic amyotrophy (Hopkins' syndrome). Muscle Nerve 1994;17:769-72.

18 Manson JI, Thong YH. Immunological abnormalities in the syndrome of poliomyelitis-like illness associated with acute bronchial asthma (Hopkins's syndrome). Arch Dis Child 1980;55:26-32.

19 Danta G. Electrophysiological study of amyotrophy associated with acute asthma (asthmatic amyotrophy). 7 Neurol Neurosurg Psychiatry 1975;38:1016-21.

20 Kyllerman MG, Herner S, Bergström TB, Ekholm SE. PCR diagnosis of primary herpesvirus type I in poliomyelitis-like paralysis and respiratory tract disease. Pediatr Neurol 1993; 9:227-9.

21 McKhann GM, Cornblath DR, Griffin JW, et al. Acute motor axonal neuropathy: a frequent cause of acute flaccid paralysis in China. Ann Neurol 1993;33 333-42. 